Tunability of the critical-current density in superconductor-ferromagnet hybrids

Nuria Del-Valle, Carles Navau, Alvaro Sanchez, and Du-Xing Chen

Citation: Appl. Phys. Lett. 98, 202506 (2011);

View online: https://doi.org/10.1063/1.3591971

View Table of Contents: http://aip.scitation.org/toc/apl/98/20

Published by the American Institute of Physics

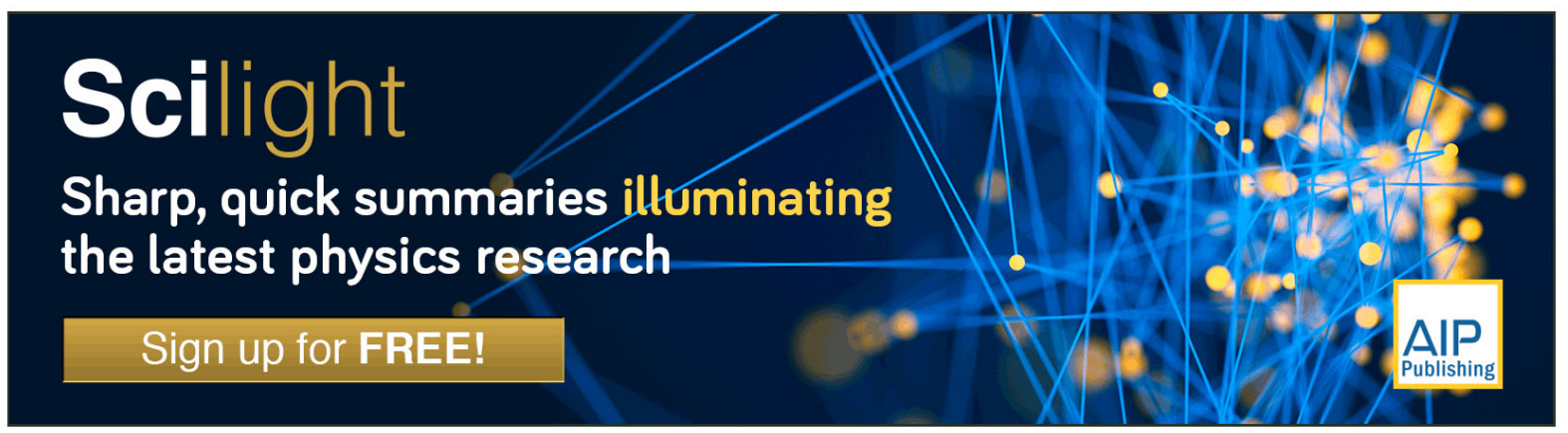




\title{
Tunability of the critical-current density in superconductor-ferromagnet hybrids
}

\author{
Nuria Del-Valle, Carles Navau, Alvaro Sanchez, a) and Du-Xing Chen \\ Department Física, Universitat Autònoma de Barcelona, Bellaterra, Barcelona, Catalonia 08193, Spain
}

(Received 1 March 2011; accepted 27 April 2011; published online 19 May 2011)

\begin{abstract}
Important modifications on the magnetization loops of the superconductor have been observed in superconductor-ferromagnet hybrids due to the effects of the ferromagnetic components, which can be used for tuning the superconductor critical-current density $J_{c}$ to desirable values. Here, a model based on an energy minimization procedure is presented to analyze the complex interaction between the superconductor and the ferromagnets. We show how the geometry and orientation of the ferromagnets can be chosen for shifting the position of the peaks appearing in the magnetization to positive or negative applied fields, and, consequently, to tune $J_{\mathrm{c}}$ in superconductor-ferromagnet hybrids. (C) 2011 American Institute of Physics. [doi:10.1063/1.3591971]
\end{abstract}

The interaction between superconductors (SCs) and ferromagnets (FMs) is a very active topic of research yielding both rich physical phenomena and promising applications. ${ }^{1-3}$ One of the lines of research that is attracting more attention is the possibility of increasing the critical-current density $J_{\mathrm{c}}$ of the SC by placing ferromagnetic components near or inside the SC. Many experiments are being carried out to study the effect of ferromagnetic parts on the $J_{\mathrm{c}}$ of the SC, both in the transport case, that is, when a current is fed in the SC via an external source, ${ }^{4-6}$ and in the magnetic case, in which current in the SC is induced by an external magnetic field $H_{\mathrm{a}}{ }^{7-10}$ Considering the magnetic case, it is known that in SCs the width of the hysteresis loop $M\left(H_{\mathrm{a}}\right)$ is directly associated to the critical-current density $J_{\mathrm{c}}\left(H_{\mathrm{a}}\right)$ of the $\mathrm{SC},{ }^{11,12}$ according to the critical-state model (CSM). ${ }^{13}$ Obtaining a desired value of $J_{\mathrm{c}}$ at a given field $H_{\mathrm{a}}$ is, therefore, equivalent to finding a hysteresis loop with the adequate shape as to yield this $J_{\mathrm{c}}$ value at $H_{\mathrm{a}}$. How to modify in general the hysteresis loop of a SC into a form corresponding to a desired value of $J_{\mathrm{c}}\left(H_{\mathrm{a}}\right)$ ? What is the effect that a given FM (e.g., a ferromagnetic film or a collection of magnetic dots) can produce in a hysteresis loop of a SC? This work aims at answering these questions. In general, the interaction of the ferromagnetic parts with the SC may occur at two different scales: at the individual vortex scale, or at that of the collective effects of many vortices. The latter situation is well described in most cases by CSM, assuming that there is a macroscopic field-dependent critical-current density; this will be the approach considered here. In this work we will present a theoretical framework and calculated results that: (i) offer a systematic understanding of the effect of a FM magnetic field in a SC $M\left(H_{\mathrm{a}}\right)$ loop; (ii) explain some anomalous experimentally measured $M\left(H_{\mathrm{a}}\right)$ loops; and (iii) predict that some other kind of anomalous $M\left(H_{\mathrm{a}}\right)$ loop until now associated to granular materials can appear in homogeneous (nongranular) materials with a particular FM configuration.

We consider hybrid SC-FM systems composed of an infinitely long type-II superconducting strip along the $x$ direction with rectangular cross-section $a_{\mathrm{SC}} \times b_{\mathrm{SC}}$ along the $y$ and $z$ directions, respectively, and, as ferromagnetic part, some

${ }^{\text {a)} E l e c t r o n i c ~ m a i l: ~ a l v a r . s a n c h e z @ u a b . e s . ~}$ permanent magnets (PMs) also infinitely long in the $x$ direction and with rectangular cross-section $a_{\mathrm{PM}} \times b_{\mathrm{PM}}$ along the $y$ and $z$ directions, respectively. The PMs are assumed to have a constant and uniform magnetization, $M_{\mathrm{PM}}$. The system is immersed in a uniform applied field of the form $\mathbf{H}_{\mathrm{a}}=H_{\mathrm{a}} \hat{\mathbf{z}}$ and we assume that the $H_{\mathrm{a}}$ value starts at zero and changes step by step along a hysteresis loop. We consider that the SC obeys the CSM with a field-dependent critical-current density $J_{\mathrm{c}}\left(H_{\mathrm{i}}\right)$, where $H_{\mathrm{i}}$ is the modulus of the total magnetic field (the sum of the applied field, the field of the PMs, and the self field created by the SC currents). The SC is cooled at zero applied field but with the magnetic field of the PMs. We assume that during the cooling process, no currents are induced in the $\mathrm{SC}^{14}$ that is, the PMs do not induce current penetration inside the SC but modify the value of $J_{\mathrm{c}}$.

The current profiles induced in the superconducting strip can be calculated by minimizing the functional given in Eq. (1) in Ref. 15, with the constraints that the net current must be zero and the current density does not exceed the $J_{\mathrm{c}}$. Introducing the $J_{\mathrm{c}}\left(H_{\mathrm{i}}\right)$ dependence by means of a first-order iterative algorithm, it is found that, following the notation of Prigozhin, ${ }^{16}$ minimizing this functional with the current density $J$ is equivalent to minimizing a functional $\mathcal{F}^{\prime}$ with the current density variation $\delta J$ defined as the difference, at present field $H$, between the present current density $J(\mathbf{r}, H)$ and the previous current density $\hat{J}(\mathbf{r}, H)$ (at the first step of $H_{\mathrm{a}}, \hat{J}(\mathbf{r}, H)$ is assumed zero). The functional $\mathcal{F}^{\prime}$ is defined as

$$
\begin{aligned}
\mathcal{F}^{\prime}[\delta J(\mathbf{r}, H)] \equiv & \int_{S} \delta J(\mathbf{r}, H)\left[\frac{1}{2} A_{\delta J}(\mathbf{r}, H)+A_{\mathrm{a}}(\mathbf{r})-\hat{A}_{\mathrm{a}}(\mathbf{r})\right. \\
& \left.+A_{\Delta \hat{J}}(\mathbf{r}, H, \hat{H})\right] \mathrm{d} S,
\end{aligned}
$$

where $S$ is the SC cross-section, $A_{\mathrm{a}}$ and $\hat{A}_{\mathrm{a}}$ are the vector potentials corresponding to the present applied field $H_{\mathrm{a}}$ and the previous applied field $\hat{H}_{\mathrm{a}}$, respectively, $A_{\delta J}$ is the vector potential created by $\delta J$, and $A_{\Delta} \hat{J}$ is the vector potential created by $\Delta \hat{J}(\mathbf{r}, H, \hat{H})$, defined as the increment between the previous current density at present field $\hat{J}(\mathbf{r}, H)$ and the previous current density at previous field, $\hat{H}, \hat{J}(\mathbf{r}, \hat{H})$. The numerical model presented here is valid for the cases where the 


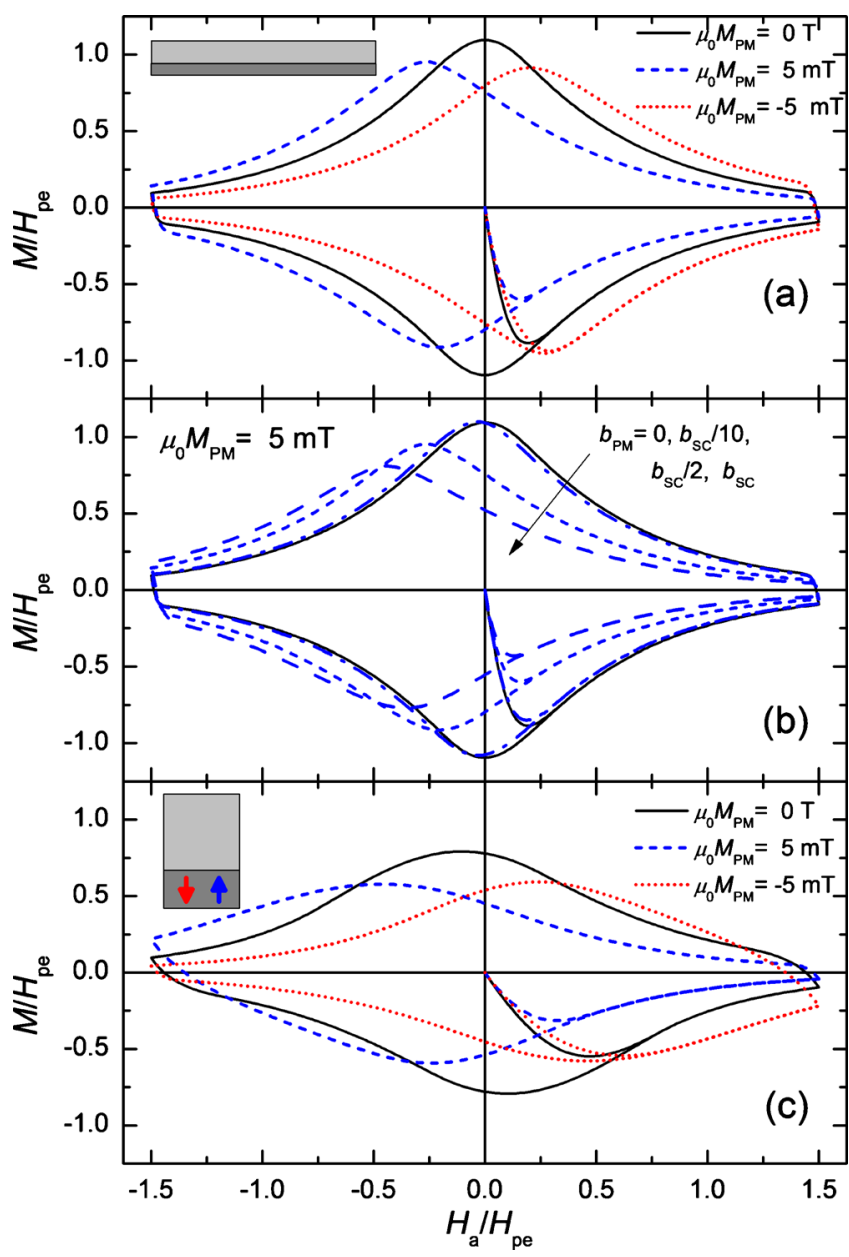

FIG. 1. (Color online) Magnetization of the SC as a function of the applied field for different SC-PM systems composed of: (a) a thin SC with $a_{\mathrm{SC}}$ $=10 \mu \mathrm{m}$ and $b_{\mathrm{SC}}=1 \mu \mathrm{m}$ on top of a thin PM of $a_{\mathrm{PM}}=10 \mu \mathrm{m}$ and $b_{\mathrm{PM}}$ $=0.5 \mu \mathrm{m}$ for different orientations of PM, (b) a thin SC with $a_{\mathrm{SC}}=10 \mu \mathrm{m}$ and $b_{\mathrm{SC}}=1 \mu \mathrm{m}$ on top of a positive PM of width $a_{\mathrm{PM}}=10 \mu \mathrm{m}$ and different thicknesses (the arrow indicates the direction of increasing $b_{\mathrm{PM}}$ ), and (c) the same as (a) but with a SC of $a_{\mathrm{SC}}=10 \mu \mathrm{m}$ and $b_{\mathrm{SC}}=10 \mu \mathrm{m}$ on top of a thick PM of $a_{\mathrm{PM}}=10 \mu \mathrm{m}$ and $b_{\mathrm{PM}}=5 \mu \mathrm{m}$. The insets of (a) and (c) show a sketch of the SC-PM systems.

components of the PM-field have the vertical and/or horizontal symmetries considered in this work. Once the current densities are calculated, the magnetization of the SC, can be calculated as $\mathbf{M}=(1 / S) \int_{S} \mathbf{r} \times \mathbf{J d S}$. Due to the geometry and unless otherwise specified, the magnetization has only $z$-component. For this work we assume an exponential dependence $J_{\mathrm{c}}\left(H_{\mathrm{i}}\right)=J_{\mathrm{c} 0} \exp \left(-\left|H_{\mathrm{i}}\right| / H_{0}\right),{ }^{17}$ with typical values $J_{\mathrm{c} 0}=4.44 \times 10^{8} \mathrm{~A} / \mathrm{m}^{2}$ and $\mu_{0} H_{0}=5.6 \times 10^{-4} \mathrm{~T}$.

In order to understand the effect of a PM in the hysteresis loop $M\left(H_{\mathrm{a}}\right)$ of a SC we first consider, in Fig. 1(a), a case of a superconducting film on top of a thin magnetic substrate. We studied the transport case for a similar hybrid SC-FM in Ref. 18 but considering the substrate as a soft FM. In Fig. 1(a), we can see the effects of having a PM substrate on the $M\left(H_{\mathrm{a}}\right)$ loop of a SC when the PM has a positive or negative vertical ( $z$ ) magnetization, as well as the loop for the isolated SC (in every loop we calculate the initial curve up to a given field $H_{\mathrm{a} \text {,max }}$, the returning curve until $-H_{\mathrm{a} \text {,max }}$, and the reverse curve to $H_{\mathrm{a} \text {,max }}$ again). ${ }^{.9}$ We observe that the peak in the returning magnetization curve occurs at $H_{\mathrm{a}} \sim 0$ for the isolated SC (as expected for a thin film ${ }^{12}$ ), whereas a positive (negative) magnetization in the PM substrate shifts the peak to the negative (positive) applied field values. The magnetization peaks in the reverse curve follow the same pattern. The peaks in $M$ appear when the average field in the SC (the applied and self fields) is similar to the average of field created by the PM (with opposite sign), so that a minimum of the average of total field is produced and $J_{\mathrm{c}}$ has a maximum. The described behavior is general but the details of the magnetization curve and the peak position change with varying the geometrical parameters. As an example, in Fig. 1(b), we show the effect of modifying the thickness of the PM film for the case of a positive PM-magnetization (with negative magnetization we obtain analogous results) and keeping the same $\mathrm{SC}$. With decreasing thickness, the field of the PM is less and compensation occurs at smaller $\left|H_{\mathrm{a}}\right|$ values, and also the height of the $M$ peak grows until approaching its value when there is no PM. Another parameter that can be modified is the thickness of the $\mathrm{SC}{ }^{20}$ Its effect on the $M\left(H_{\mathrm{a}}\right)$ loop is illustrated in Fig. 1(c) for the case of a SC with square crosssection on top of a thick magnetic substrate. In this case, the loop with the isolated SC shows a magnetization peak at negative $H_{\mathrm{a}}$ values [as is known to occur for not very thin samples according to CSM (Ref. 12)], and the effect of the PM-field yields similar effects as for films [Fig. 1(a)] but with wider peaks. The reason for this widening is that the SC volume is now larger and the field in its interior is more inhomogeneous so field compensation occurs more gradually.

This seems to be general behavior of the effect of a PM in the hysteresis loop of a SC: instead of having a peak at negative $H_{\mathrm{a}}$ fields in the reverse curve and one at positive fields in the returning curve (as in standard CSM), both peaks are shifted to one side because of field compensations. Thus, our calculations explain the asymmetry observed in several magnetization loops of SCs with periodic arrays of magnetic dots or antidots. ${ }^{1}$ A particularly interesting experiment performed in Ref. 7 showed a hysteresis loop of a superconducting $\mathrm{Pb}$ film grown on an array of $\mathrm{Co} / \mathrm{Pt}$ magnetic dots, with the general characteristics described in Fig. 1. In that work, the peaks in the magnetization occurred at a positive $H_{\mathrm{a}}$ field when the dots were magnetized in the positive direction (opposite to the results in Fig. 1). This can be understood from the fact that in their case of sparsely distributed magnetic dots the main contribution of the dots magnetic field to compensation is not on the areas on top of the dots (as considered in Fig. 1) but in the space between them, where the dots field has the opposite polarity. ${ }^{21,22}$ Although the geometry of our simulated system is not exactly the same as in the experimental case (we consider infinitely long PMs instead of an array of magnetic dots and only consider a fragment of SC on top of two PMs) when taking into account a equivalent space between dots with reversed field, we show in Fig. 2 a calculated $M\left(H_{\mathrm{a}}\right)$ that reproduce well the experiments (Fig. 1 in Ref. 7), including the sign of the field.

At this point we can ask whether the described behavior is the only possible effect that a PM can create on the hysteresis loop of a SC. Or its equivalent question, can $J_{\mathrm{c}}$ be tuned only in this way? We will show that other interesting shapes of the loop can indeed be obtained, to be confirmed by future experiments. We now consider a PM inserted inside the SC and having horizontal (y) magnetization. In this case, the magnetization of the SC also has $y$-component, as 


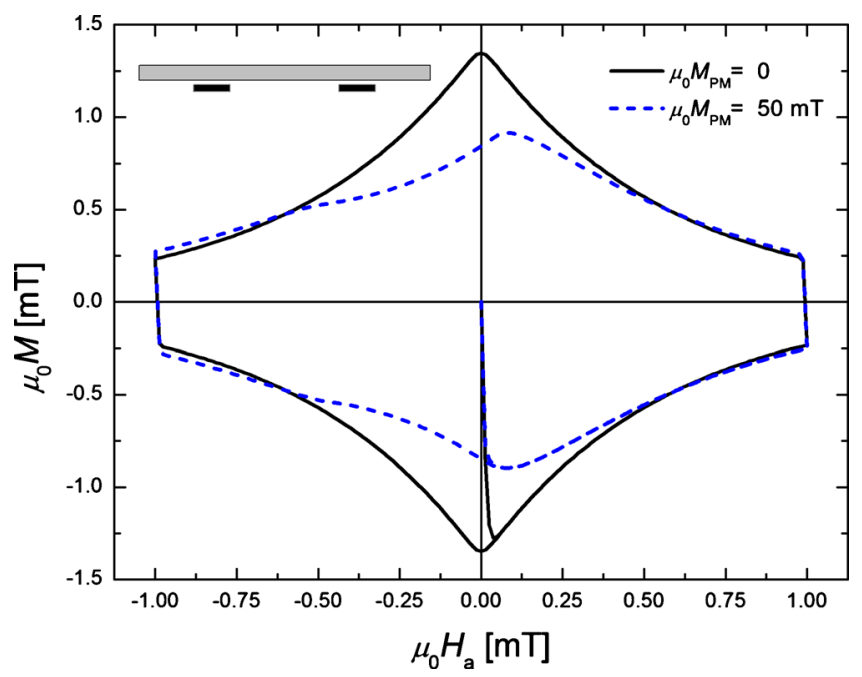

FIG. 2. (Color online) Magnetization of the SC as a function of the applied field for the case of a SC-PM system composed of a thin SC of dimensions $a_{\mathrm{SC}}=10 \mu \mathrm{m}$ and $b_{\mathrm{SC}}=100 \mu \mathrm{nm}$ on top of a set of two identical PMs of dimensions $a_{\mathrm{PM}}=1.25 \mu \mathrm{m}$ and $b_{\mathrm{PM}}=20 \mathrm{~nm}$ and with a lateral distance between them of $d_{\mathrm{PM}}=3.75 \mu \mathrm{m}$. The SC and PMs are vertically separated a distance $d=5 \mathrm{~nm}$. The inset shows a sketch of the SC-PMs system.

shown by the antisymmetry of the current density profiles of the inset of Fig. 3 but very small (at most few percent) compared to the $z$-component. The calculated vertical $M\left(H_{\mathrm{a}}\right)$ plotted in Fig. 3 shows an interesting shape: the peak in the reverse magnetization curve is at a positive $H_{\mathrm{a}}$ and that at the returning curve is at a negative $H_{\mathrm{a}}$. This behavior has been found experimentally in cases in which the SC was granular and was associated to this granularity. ${ }^{23,24}$ With the PM used in Fig. 3, unlike the cases of Fig. 1, the z-component of the PM-field is antisymmetric with respect to both vertical and horizontal axes, and, as the applied field is uniform, the cancellation between the PM and applied fields cannot be the reason for the peak. Instead, the $z$-component of the self field

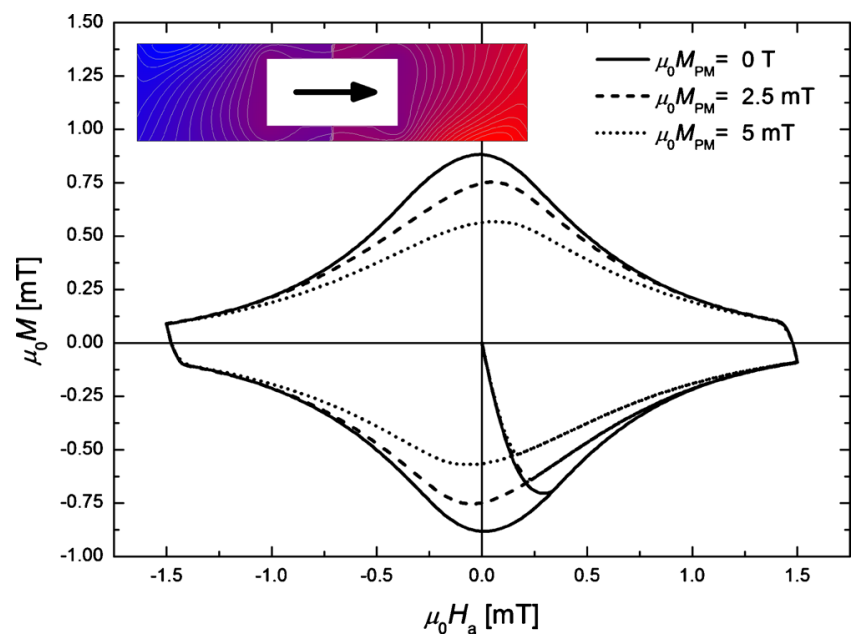

FIG. 3. (Color online) Magnetization of the SC as a function of the applied field for the case of a SC-PM system composed of a SC with dimensions $a_{\mathrm{SC}}=10 \mu \mathrm{m}$ and $b_{\mathrm{SC}}=2.5 \mu \mathrm{m}$ inside of which there is a centered PM of dimensions $a_{\mathrm{PM}}=10 / 3 \mu \mathrm{m}$ and $b_{\mathrm{PM}}=5 / 3 \mu \mathrm{m}$. The PM has different positive horizontal magnetizations. The solid line corresponds to the case of the isolated SC but with a hole inside (null PM). The inset shows the criticalcurrent density profile when the reverse magnetization curve has a peak for the case of $5 \mathrm{mT}$. The right (left) zones indicates negative (positive) current densities and the central zone corresponds to the PM. The darkest zones indicate the largest absolute values of the current density. of the SC (mostly positive in the reverse curve and small) is the responsible that the reverse curve has a peak at positive and small applied fields, since there the sum of applied and vertical self fields compensates, on average, the $z$-component of the PM-field, and $J_{c}$ has a maximum. Thus, we theoretically predict here that such anomalous shape can arise in homogeneous SCs, so our results should be taken into account when studying granularity in SCs with FMs.

In conclusion, we have theoretically explained a set of recently published experimental data on the effect of a PM (or an array of dots) in the magnetization and critical-current of superconducting films. A continuous PM film and an array of magnetic dots may yield a different behavior, including a change in sign in the effect. Some anomalous hysteresis loops found until now only in granular system have been shown to appear also in homogeneous SCs with FMs.

We thank Consolider Project NANOSELECT (Grant No. CSD2007-00041) for financial support.

${ }^{1}$ A. Y. Aladyshkin, A. V. Silhanek, W. Gillijns, and V. V. Moshchalkov, Supercond. Sci. Technol. 22, 053001 (2009).

${ }^{2}$ M. Velez, J. I. Martin, J. E. Villegas, A. Hoffmann, E. M. Gonzalez, J. L. Vicent, and I. K. Schuller, J. Magn. Magn. Mater. 320, 2547 (2008).

${ }^{3}$ S. C. Wimbush, J. H. Durrell, R. Bali, R. Yu, H. Y. Wang, S. A. Harrington, and J. L. MacManus-Driscoll, IEEE Trans. Appl. Supercond. 19, 3148 (2009).

${ }^{4}$ N. Touitou, P. Bernstein, J. F. Hamet, Ch. Simon, L. Mechin, J. P. Contour, and E. Jacquet, Appl. Phys. Lett. 85, 1742 (2004).

${ }^{5}$ M. Morelle and V. V. Moshchalkov, Appl. Phys. Lett. 88, 172507 (2006).

${ }^{6}$ D. Y. Vodolazov, B. A. Gribkov, A. Y. Klimov, V. V. Rogov, and S. N. Vdovichev, Appl. Phys. Lett. 94, 012508 (2009).

${ }^{7}$ D. G. Gheorghe, R. J. Wijngaarden, W. Gillijns, A. V. Silhanek, and V. V. Moshchalkov, Phys. Rev. B 77, 054502 (2008).

${ }^{8}$ D. J. Morgan and J. B. Ketterson, Phys. Rev. Lett. 80, 3614 (1998).

${ }^{9}$ M. Lange, M. J. Van Bael, A. V. Silhanek, and V. V. Moshchalkov, Phys. Rev. B 72, 052507 (2005).

${ }^{10}$ M. D. Sumption, E. W. Collings, and P. N. Barnes, Supercond. Sci. Technol. 18, 122 (2005).

${ }^{11}$ D.-X. Chen and R. B. Goldfarb, J. Appl. Phys. 66, 2489 (1989).

${ }^{12}$ A. Sanchez and C. Navau, Supercond. Sci. Technol. 14, 444 (2001).

${ }^{13}$ C. P. Bean, Phys. Rev. Lett. 8, 250 (1962).

${ }^{14}$ N. Del-Valle, A. Sanchez, E. Pardo, D.-X. Chen, and C. Navau, Appl. Phys. Lett. 91, 112507 (2007).

${ }^{15}$ A. Sanchez, N. Del-Valle, E. Pardo, D.-X. Chen, and C. Navau, J. Appl Phys. 99, 113904 (2006).

${ }^{16}$ L. Prigozhin, IEEE Trans. Appl. Supercond. 7, 3866 (1997).

${ }^{17}$ D.-X. Chen, A. Sanchez, and J. S. Muñoz, J. Appl. Phys. 67, 3430 (1990).

${ }^{18}$ A. Sanchez, N. Del-Valle, C. Navau, and D.-X. Chen, Appl. Phys. Lett. 97, 072504 (2010).

${ }^{19} \mathrm{M}$ and $H_{\mathrm{a}}$ in Fig. 1 are normalized to $H_{\mathrm{pe}}$ (defined as the field of total penetration in an infinite samples) because in this way the curve for the SC does not depend on particular dimensions (Refs. 12 and 17). However, the loops when there is a PM depend also on $M_{\mathrm{PM}}$ and the width $a_{\mathrm{SC}}$. In our case, the chosen values of $J_{\mathrm{c} 0}$ and $H_{0}$ are equivalent to consider $p$ $=J_{\mathrm{c} 0} a_{\mathrm{SC}} / 2 H_{0}=5$, with $a_{\mathrm{SC}}=10 \mu \mathrm{m}$, which describes the strength of the exponential decay of $J_{\mathrm{c}}$ with $H_{\mathrm{i}}$, and $\mu_{0} H_{\mathrm{pe}}=\mu_{0} H_{0} \ln (1+p)=1 \mathrm{mT}$.

${ }^{20}$ A. Sanchez, C. Navau, N. Del Valle, D.-X. Chen, and J. R. Clem, Appl. Phys. Lett. 96, 072510 (2010).

${ }^{21}$ M. Lange, M. J. Van Bael, Y. Bruynseraede, and V. V. Moshchalkov, Phys. Rev. Lett. 90, 197006 (2003).

${ }^{22}$ K. Kim, A. E. Ozmetin, D. G. Naugle, and I. F. Lyuksyutov, Appl. Phys. Lett. 97, 042501 (2010).

${ }^{23}$ A. Palau, T. Puig, X. Obradors, E. Pardo, C. Navau, A. Sanchez, A. Usoskin, H. C. Freyhardt, L. Fernandez, B. Holzapfel, and R. Feenstra, Appl. Phys. Lett. 84, 230 (2004).

${ }^{24}$ J. Yoo, S. M. Lee, Y. H. Jung, J. Lee, D. N. Xuan, D. Youm, H. Kim, H. Ha, K. J. Song, S. S. Oh, and S. Oh, Supercond. Sci. Technol. 21, 085020 (2008). 